International Tinnitus Journal. 2019;23(1):37-41.

\title{
Perceptual organization of sequential stimuli in cochlear implant listeners: A temporal processing approach
}

Nader Saki ${ }^{1}$

Soheila Nikakhlagh ${ }^{1}$

Golshan Mirmomeni²

Arash Bayat ${ }^{3 *}$

\begin{abstract}
Objective: Cochlear Implant $(\mathrm{Cl})$ users often suffer difficulties in perceiving speech in noisy environments that could be attributed to reduced Auditory Stream Segregation (ASS) ability. ASS is the process used to separate a complex sound into different perceptual streams. The evidence that $\mathrm{Cl}$ listeners routinely experience stream segregation skill is limited and equivocal. The present study was aimed to investigate the effects of temporal cues on ASS performance in postlingually deaf listeners with $\mathrm{Cl}$.

Methods: Nineteen (age range: 28-64 years old) monaurally cochlear implanted listener participated in this study. They were presented with 30 -s sequences of alternating stimuli in a repeating A-B-A-A-B-A....sequence, where "tone A" corresponds to a stimulus applied to electrode 11, and "tone B" to a stimulus on one of the other electrode. To investigate the effect of temporal cues on ASS, four different tone repetition times (TRTs) were utilized: 50, 100, 150, and $200 \mathrm{~ms}$. Speech discrimination scores in noise were also recorded for every $\mathrm{Cl}$ recipients

Results: Only 6 (32\%) Cl users demonstrated ASS pattern similar to the normal hearing subjects, while the majority of the users $(n=13)$ possessed poorer ASS skills. An analysis of variance showed a significant effect of electrode separation $(p<0.001)$ and TRT $(p=0.041)$, but there was no significant interaction between electrode separation and TRT variables. The best ASS performance was obtained when TRT was $200 \mathrm{~ms}$, and there was no significant effect for other TRT conditions. Moderate, significant correlations between streaming and speech discrimination measurement in noise was also observed $(r=0.62)$, with better stream segregation associated with better understanding of speech in noise.
\end{abstract}

Conclusion: ASS is a contributing factor in the ability to perceive speech in background noise. The inability of some $\mathrm{Cl}$ recipients to perform stream segregation may therefore contribute to their difficulties in noisy backgrounds. Furthermore, stream segregation ability is related to the tone repetition time between the sounds.

Keywords: Auditory stream segregation; cochlear implant; adult temporal cure.

${ }^{1}$ Hearing Research Center, Imam Khomeini Hospital, Ahvaz Jundishapur University of Medical Sciences, Ahvaz, Iran

${ }^{2}$ Department of Statistics and Epidemiology, School of Health, Ahvaz Jundishapur University of Medical Sciences, Ahvaz, Iran

${ }^{3}$ Musculoskeletal Rehabilitation Research Center, School of Rehabilitation, Ahvaz Jundishapur University of Medical Sciences, Ahvaz, Iran

*Send correspondence to:

Arash Bayat

Musculoskeletal Rehabilitation Research Center, School of Rehabilitation, Ahvaz Jundishapur University of Medical Sciences, Ahvaz, Iran. E-mail:bayat-a@ajums.ac.ir

Phone:+009-8939-440-2323

Paper submitted to the ITJ-EM (Editorial Manager System) on April 17, 2019; and accepted on May 16, 2019. 


\section{INTRODUCTION}

Effective anatomical and functional integration of the peripheral and central auditory system and an acoustically appropriate communication context are necessary for appropriate understanding of speech. The presence of noise, which may decrease the probability of acoustic information being available. Noise affects understanding of speech in any individual even the healthy ones. This issue is aggregated in hearing impaired individuals when speech and noise compete at the same time resulting in the loss of acoustic information. This problem may be partly due to reduced audibility. However, some difficulty persists even when sounds are amplified to a comfort level ${ }^{1-3}$. It has been reported that some supra-threshold auditory capacities may be affected in these populations ${ }^{3-6}$. Loss of acoustic information could be compensated using other non-auditory cues during silence. One of these mechanisms utilized by auditory system is Auditory Stream Segregation (ASS). ASS as a fundamental aspect of hearing and speech perception is the perceptual grouping of sounds, to form coherent representations of objects in the acoustic scene. Cochlea acts like a bank of parallel band-pass filters through which partitions the spectrum of incoming sound into several frequency bands. When we listen to rapid alternating sequences of sounds, such as those that occur in speech or music, sounds with the components composed of similar frequency ranges strongly interact with each other and tend to be grouped together so that they are perceived as if they come from a single source. This phenomenon is called fusion. On the contrary, sounds with components in different frequency ranges tend to be perceived as different streams as if they are coming from more than one source, the phenomenon that called segregation) $)^{7-9}$. Mackersie ${ }^{10}$ suggested that stream segregation by hearing-impaired listeners are originated from their ability to perceptually separate competing, concurrent sentences spoken by talkers of different genders. It is of considerable interest, therefore, to discover whether $\mathrm{Cl}$ listeners are capable of Auditory Stream Segregation (ASS). Cochlear Implant (Cl) listeners rely on the electrical signals encoding information in the acoustical signals to stimulate the auditory nerve to form an auditory perception. All the incoming sounds are processed through the processor according to some programmed rules $^{11-13}$. Measures of auditory stream segregation among $\mathrm{Cl}$ patients yield conflicting findings. Hong and Turner ${ }^{14}$ reported that $\mathrm{Cl}$ users can form perceptual segregation but with reduced capability; whereas, Cooper and Roberts ${ }^{15}$ argued for the opposite view. Half of the $\mathrm{Cl}$ users in Hong and Turner ${ }^{14}$ study performed within the range of the normally hearing subjects, and the other half showed less obligatory streaming. However, Cooper and Roberts ${ }^{15}$ asked implanted patients to show whether alternating stimulation on two electrodes (ABA-ABA pattern) was perceived as integrated or segregated. They also reported that $\mathrm{Cl}$ recipients show little or no evidence of automatic stream segregation. The present study aimed to measure the role of temporal cues on ASS ability in post-lingual $\mathrm{Cl}$ listeners.

\section{MATERIALS AND METHODS}

\section{Participants}

Nineteen postlingually deaf listeners with Cls participated in this study. All of the participants were users of Nucleus device (Sydney, Australia) which was set to their recommended settings during the experiment and had at least 2 years of experience with their devices at the time of the experiments (Table 1). Prior to the start of the experiments, all listeners provided written informed consent on their participation in the study. All

Table 1. Demographic and clinical details of the cochlear implant listeners.

\begin{tabular}{|c|c|c|c|c|c|}
\hline Subject & Gender & Age & $\begin{array}{l}\text { Open set speech } \\
\text { recognition score (\%) }\end{array}$ & $\mathrm{Cl}$ experience (years) & Side of implantation \\
\hline $\mathrm{Cl} 1$ & $\mathrm{M}$ & 49 & 68 & 3 & Right \\
\hline $\mathrm{Cl} 2$ & $\mathrm{~F}$ & 42 & 78 & 4.5 & Right \\
\hline $\mathrm{Cl} 3$ & $\mathrm{M}$ & 56 & 66 & 2 & Left \\
\hline $\mathrm{Cl} 4$ & $\mathrm{M}$ & 44 & 92 & 3 & Right \\
\hline $\mathrm{Cl} 5$ & $\mathrm{~F}$ & 20 & 74 & 16 & Left \\
\hline $\mathrm{Cl} 6$ & $\mathrm{~F}$ & 52 & 86 & 4 & Left \\
\hline $\mathrm{Cl} 7$ & $M$ & 46 & 88 & 2.5 & Right \\
\hline $\mathrm{Cl} 8$ & $\mathrm{~F}$ & 36 & 66 & 12 & Left \\
\hline $\mathrm{Cl} 9$ & $\mathrm{~F}$ & 28 & 72 & 7 & Right \\
\hline $\mathrm{Cl} 10$ & M & 33 & 88 & 13 & Right \\
\hline $\mathrm{Cl} 11$ & $\mathrm{~F}$ & 48 & 76 & 6 & Right \\
\hline $\mathrm{Cl} 12$ & $\mathrm{~F}$ & 44 & 92 & 15 & Right \\
\hline $\mathrm{Cl} 13$ & $M$ & 38 & 96 & 3.5 & Left \\
\hline Cl 14 & $\mathrm{~F}$ & 64 & 84 & 6 & Left \\
\hline Cl 15 & $\mathrm{~F}$ & 51 & 76 & 8 & Right \\
\hline $\mathrm{Cl} 16$ & $\mathrm{~F}$ & 64 & 56 & 5 & Right \\
\hline $\mathrm{Cl} 17$ & $\mathrm{~F}$ & 38 & 80 & 6 & Left \\
\hline Cl 18 & $M$ & 32 & 62 & 9 & Right \\
\hline Cl 19 & $\mathrm{~N}$ & 43 & 70 & 14 & Right \\
\hline
\end{tabular}


experimental procedures were approved by the local Ethics Committee of AJUMS, Ahvaz, Iran (Registration code: HRC-9410) which were in complete accordance with the ethical regulations of human studies set by the Helsinki declaration (2014).

\section{ASS assessment}

In this study, we used a stimulus paradigm based on the one introduced by Cooper and Roberts ${ }^{15}$ to evaluate auditory stream segregation ability. In cochlear implant device, electrodes are numbered 1 to 22 from base to apex; i.e., lower numbers correspond to higher frequency areas according to normal tonotopic organization. The listeners were presented with 30 -s sequences of alternating stimuli in a repeating $A-B-A-A-B-A$..sequence, where $A$ corresponds to a stimulus applied to electrode 11 (in the middle of the electrode array, corresponding to a pure tone at $1938 \mathrm{~Hz}$ ) and B corresponds to a stimulus on one of the other electrodes, i.e., 1 to 10 and 12 to 22. The electrode utilized for tone $B$ was chosen at random order between presentation sequences, but did not change within each 30-s sequence. In total, four repetitions of each $A B A$ combination were used. In order to investigate the effect of temporal characteristics on ASS, four different tone repetition times were used: 50, 100, 150, and $200 \mathrm{~ms}$. Sound stimuli were introduced to the $\mathrm{Cl}$ listeners in a free-field condition in a doublewalled sound attenuated booth through a single, frontally located loudspeaker. Stimuli were generated using a MATLAB script at a sampling rate of $22,050 \mathrm{~Hz}$. The 4th order Butterworth filters were designed and applied to the stimulus in the Analog Filter Design (AFD) Toolbox in MATLAB. Each listener was seated in front of a laptop. They were instructed to press two different keys when they perceive a sequence as an 'integrated' (1 stream) or 'segregated' (2 streams). In the initial phases, the subject could hear two streams as the frequency separation was set sufficiently large to elicit the perception of segregation. Then the frequency separation between tone $A$ and tone $B$ gradually reduced until the subject reported hearing one stream (integrating $A$ and $B$ tones).

\section{Speech recognition test}

The speech material consisted of phonetically balanced 50-element lists of Persian nonsense words. Four nonsense word lists were utilized in the experiment during which 200 different stimuli were used. The words were processed offline in MATLAB software by the proposed algorithm and presented to the subjects at a comfortable rate. Sentences were presented to the listeners in blocks, with about 200 nonsense words per session. Participants were instructed to repeat the words they heard.

\section{Statistical analysis}

All statistical analyses of this study were performed with statistical package of SPSS (IBM Corporation, New York, USA, and Version 21). An analysis of variance (ANOVA) test with the within-subject factor of "time" and the between-subject factor of the assessed measures was performed for evaluating changes in the study variables. For the ANOVA tests, the sphericity was evaluated using the Mauchly's test, and in case of a violation of sphericity, the Greenhouse-Geisser correction was applied. The 0.05 level of probability was used as the criterion of statistical significance.

\section{RESULTS}

The $\mathrm{Cl}$ subjects were 19 postlingually deafened adults (11 females, 8 males) who had full electrode insertions. Table 1 shows the demographic information and clinical factors of the patients. Our results indicated that the ASS skills of the $\mathrm{Cl}$ listeners significantly varied: $32 \%(n=6)$ of patients demonstrated clear skill comparable to the normal hearing subjects while $52 \%(n=10)$ of them showed reduced skills. ASS skills was seriously affected in $16 \%$ $(n=3)$ of participants. Figure 1 shows the mean perceived segregation reported for each electrode for tone $B$ in different TRTs. Our results indicated an obvious effect of electrode number on auditory streaming performance that was symmetrical against the electrode 11. The proportion of time that patients reported hearing a segregated percept improved as the spatial separation between tone $A$ and tone $B$ increased in terms of electrode number and the lowest reported segregation was observed when tone B was on electrode 10 or 12. An ANOVA analysis showed a significant effect of electrode separation $(p<0.001)$ and TRT $(p=0.041)$, but there was no significant interaction between electrode separation and TRT variables. Pairwise comparisons showed that the best ASS performance was observed when TRT value was $200 \mathrm{~ms}$ and there was no significant effect for other TRT conditions (50, 100 and $150 \mathrm{~ms}$ ) ( $p>0.05)$. Moreover, we observed a moderate correlation between the perceptual segregation and the

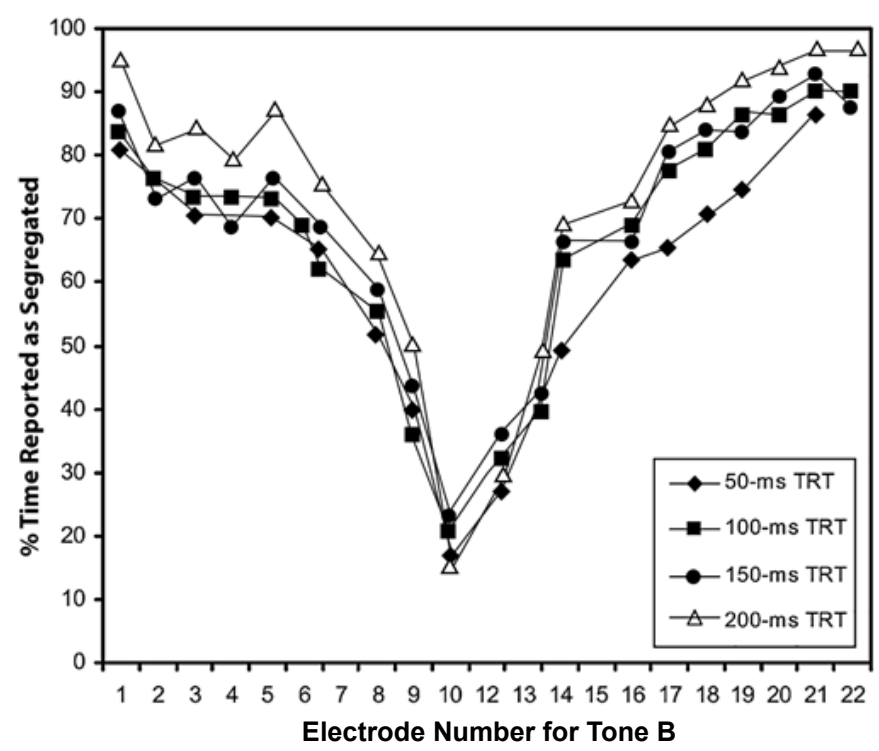

Figure 1. The mean auditory stream segregation ability (in percentage) for cochlear implant listeners in different Tone Repetition Times (TRTs). Higher numbers denote more apically placed electrodes. 
speech discrimination ability from the background noise (Pearson's coefficient: $0.62 ; p<0001$ ) so that higher ability in ASS is associated with better understanding of speech in noisy backgrounds.

\section{DISCUSSION}

Cochlear implants extract the temporal information from the incoming sounds and impose them upon the electrical pulses. Therefore, the temporal cues are an important factor for $\mathrm{Cl}$ listeners to comprehend sounds. If $\mathrm{Cl}$ users be able to segregate sound waves according to the temporal cues as normal-hearing subjects do, their reduced speech understanding ability in noisy situations may be partially due to their segregation capability. The role of temporal factors on ASS in Cls has been evaluated in different studies. The findings of these studies suggest that large differences in the amplitude modulation or the pulse rate between the $A$ and the $B$ sounds facilitate both schema-based ${ }^{14-16}$ and obligatory ${ }^{17}$ stream segregation. Paredes-Gallardo et al. ${ }^{11}$ investigated the effect of temporal cues on schema-based ASS in $\mathrm{Cl}$ listeners using a delay detection task composed of a sequence of bursts of pulses $(B)$ on a single electrode interleaved with a second sequence $(A)$ presented on the same electrode with a different pulse rate. Their results demonstrated that $\mathrm{Cl}$ patients can make use of temporal information to segregate sound waves when their attention is directed toward segregation. Furthermore, they reported a buildup process similar to that observed in normally hearing listeners. The similarity between the findings for $\mathrm{Cl}$ users and those reported for normally hearing listeners could be attributed to a similar underlying mechanism for ASS in both groups ${ }^{11}$. Our findings showed that the stream segregation skills of the $\mathrm{Cl}$ listeners significantly varied: only $32 \%$ of the listeners indicated clear streaming skill, while the other showed little or remarkable reduction in ASS ability. The heterogeneity observed in this study may be attributed to different factors such as etiology of hearing loss, the duration of $\mathrm{Cl}$ usage, and rehabilitation history. It seems that for a fixed frequency separation, a smaller value of TRT (faster rate) is associated with an increased tendency towards stream segregation. Cooper and Roberts evaluated 8 postlingually deafened adults who were experienced users of $\mathrm{Cl}$ devices ${ }^{15}$. Their findings showed that although there were discrepancies in reported segregation ability for different TRTs at larger electrode separations, but that there was no significant influence of varying TRT towards the apical or basal ends of the electrode array. However, our findings indicated that the ASS performance will be improved when TRT value was $200 \mathrm{~ms}$ and for smaller values of TRT, its effect was not significant. In addition, our results showed a moderate correlation between the measure of perceptual segregation and the speech recognition ability in background noise, with better stream segregation associated with better understanding of speech in noise. Consistent with our results, Hong and Turner reported a moderate correlation between the ASS performance and speech recognition ability in $\mathrm{Cl}$ listeners ${ }^{14}$. They evaluated the ASS ability of the $\mathrm{Cl}$ listeners through choosing three pure tone sequences $-200,800$, and $2000 \mathrm{~Hz}$ for tone $A$. The tone $B$ was varied systematically by a fraction of an octave from the tone $A$ of a particular base frequency. They found a significant correlation between speech recognition and segregation performance for higher frequencies, although no statistically significant association was reported for the $200 \mathrm{~Hz}$. However, the large individual variability in $\mathrm{Cl}$ users speech processing strategies and processor parameters, makes stimulus control difficult in such experiments. Chatterjee et al. ${ }^{16}$ utilized Amplitude Modulation (AM) as an effective acoustic cue for stream segregation in cochlear implant and simulation listeners. They concluded that the only listener that revealed definite stream segregation is an experienced user and shows high speech perception skills.

\section{CONCLUSION}

The ASS is a contributing factor in the ability to understand speech in background noise. The inability of some $\mathrm{Cl}$ recipients to perform stream segregation may therefore contribute to their difficulties in noise backgrounds. Furthermore, stream segregation ability is related to the tone repetition time between the sounds.

\section{CONFLICT OF INTEREST}

The author declares no potential conflict of interest on publishing this paper

\section{REFERENCES}

1. Moore BC. Cochlear hearing loss: physiological, psychological and technical issues. 2nd Edition; Wiley-Interscience Publisher. 2007:51-63

2. Moore BC. Rose MM. Perceptual grouping of tone sequences by normally hearing and hearing-impaired listeners. J Acoust Soc Am. 1997;102(3):1768-78.

3. Rose MM, Moore BC. The relationship between stream segregation and frequency discrimination in normally hearing and hearingimpaired subjects. Hear Res. 2005;204:16-28.

4. Oxenham AJ. Pitch perception and auditory stream segregation: implications for hearing loss and cochlear implants. Trends Amplif. 2008;12(4):316-31.

5. Bayat A, Farhadi $M$, Pourbakht $A$, Sadjedi $H$, Emamdjomeh $H$, Mirmomeni G. A comparison of auditory perception in hearingimpaired and normal-hearing listeners: an auditory scene analysis study. Iran Red Crescent Med J. 2013;15(11):e9477.

6. Chatterjee M, Galvin JJ. Auditory streaming in cochlear implant listeners. J Acoust Soc Am. 2002;111:24-9.

7. Nikakhlagh S, Yadollahpour A, Karimi M, Bagheripour H, Hematipour $S$, Malehi AS, et al. Investigating gender differences on the age of suspicion of children with hearing loss in Iran. Int $\mathrm{J}$ Ment Health Addict. 2017;15:271-6.

8. Bregman AS. Auditory scene analysis: the perceptual organization of sound. First edition. MIT Press Publisher. 1990.

9. Barniv D, Nelken I. Auditory streaming as an online classification process with evidence accumulation. PLoS One. 2015;10(12): e0144788. 
10. Mackersie CL. Talker separation and sequential stream segregation in listeners with hearing loss: patterns associated with talker gender. J Speech Lang Hear Res. 2003;46:912-8.

11. Paredes-Gallardo A, Madsen SMK, Dau T, Marozeau J. The role of temporal cues in voluntary stream segregation for cochlear implant users. Trends Hear. 2018;22:2331216518773226.

12. Böckmann-Barthel M, Deike S, Brechmann A, Ziese M, Verhey JL. Time course of auditory streaming: do $\mathrm{Cl}$ users differ from normalhearing listener?, Front Psychol. 2014;5:775.

13. Paredes-Gallardo A, Madsen SMK, Dau T, Marozeau J. The role of place cues in voluntary stream segregation for cochlear implant users. Trends Hear. 2018;22:2331216517750262.
14. Hong RS, Turner CW. Pure-tone auditory stream segregation and speech perception in noise in cochlear implant recipients. J Acoust Soc Am. 2006;120:360-74.

15. Cooper HR, Roberts B. Auditory stream segregation of tone sequences in cochlear implant listeners. Hear Res. 2007;225:11 24.

16. Chatterjee M, Sarampalis A, Oba SI. Auditory stream segregation with cochlear implants: A preliminary report. Hear Res. 2006;222:100-7.

17. Duran SI, Collins LM, Throckmorton CS. Stream segregation on a single electrode as a function of pulse rate in cochlear implant listeners. J Acoust Soc Am. 2006;132:3849-55. 